\title{
Statistical Displacement Analysis for Handwriting Verification
}

\author{
Yoshiki Mizukami ${ }^{1}$, Katsumi Tadamura ${ }^{1}$, \\ Mitsu Yoshimura ${ }^{2}$, and Isao Yoshimura ${ }^{3}$ \\ 1 Yamaguchi University \\ 2 Ritsumeikan University \\ 3 Tokyo Science University
}

\begin{abstract}
In this paper, it is assumed that each writer has his or her own statistics of handwriting displacement, therefore a statistical displacement analysis for handwriting verification is proposed. Here, a regularization method with the coarse-to-fine strategy computes the displacement function in questionable handwritten letters, and then it is normalized to remove the noisy displacement that arises from the position drift and scaling variation. Finally, the normalized displacement function and the statistics of displacement obtained in advance from registered authentic letters are used to calculate the distance from a standard handwritten letter to a questionable one. A fundamental simulation was conducted in order to evaluate the performance of the proposed method.
\end{abstract}

\section{Introduction}

Handwriting is one kind of biometrics and has played a major role in proving documentary evidence. As shown in the review of Plamondon et al. 1, many researchers are involved with this topic. Until now, several groups have tried to measure distance or similarities between a registered signature and a questionable signature after computing the displacement contained in the questionable one. For instance, de Bruyne and Forre 2 devised a linear deformation method for signature verification and Naske 3 suggested a block matching method. Mizukami et al. 4] computed the non-linear displacement in questionable signatures based on March's regularization algorithm [5, 6], which has been successfully applied to handwritten character recognition 7 .

After reviewing previous attempts, it was noted that most of the researchers and engineers have been computing a displacement in order to eliminate it, while human handwriting experts are focusing on analyzing the tendency of the displacement. In this study, therefore, we propose a statistical displacement analysis method for handwriting verification based on an assumption that each writer has his or her own statistics of handwriting displacement, such as its average and variance of displacement. The following sections describe the details of the proposed method, the simulation results and the conclusion. 


\section{Proposed Method}

In the field of computer vision, March [6] suggested a regularization method for acquiring disparity in two stereo images. This study utilizes March's method and the coarse-to-fine strategy together to compute 2-dimentional displacements in questionable handwriting [4, normalizes the obtained displacement, and computes the distance based on the statistics of displacement to verify the questionable handwriting (See Fig. 1). In this section, first the computation of displacement with the coarse-to-fine strategy is described, and then the procedures for normalizing the computed displacement and measuring the distance are explained.

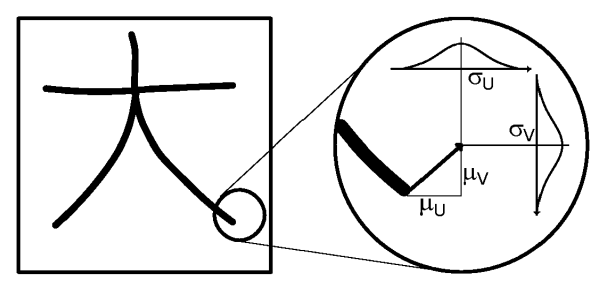

Fig. 1. statistics of displacement in handwriting

\subsection{Displacement Computation Based on Coarse-to-Fine Strategy}

The computation procedure of optimum displacement field between two isolated handwritten letters is described here. A handwritten letter is denoted as a binary image $f(x, y)$, where $1 \leq x \leq n_{x}$ and $1 \leq y \leq n_{y}$, another handwritten letter as $g(x, y)$. In the framework of regularization theory [5], the optimum displacement is given as a 2-dimensional functions $(u(x, y), v(x, y))$, which minimize the following functional $E(u, v)$,

$$
\begin{gathered}
E(u, v)=P(u, v)+\lambda S(u, v) \\
P(u, v)=\iint\{f(x+u(x, y), y+v(x, y))-g(x, y)\}^{2} d x d y \\
S(u, v)=\iint\left\{\left(\frac{\partial u}{\partial x}\right)^{2}+\left(\frac{\partial u}{\partial y}\right)^{2}+\left(\frac{\partial v}{\partial x}\right)^{2}+\left(\frac{\partial v}{\partial y}\right)^{2}\right\} d x d y
\end{gathered}
$$

where the functional $P(u, v)$ represents the difference between $f$ and $g$ with taking into account the displacement function $(u, v)$, the functional $S(u, v)$ represents the penalty of departure from smoothness in $(u, v)$ and $\lambda$ is a regularization parameter balancing them 8 . According to calculus of variation, the following Gauss-Seidel iterative equations give the optimum displacement function $(u, v)$,

$$
\begin{aligned}
u^{[t+1]}(x, y)=\bar{u}^{[t]} & (x, y)-\frac{1}{4 \lambda} \frac{\partial f\left(x+\bar{u}^{[t]}(x, y), y+\bar{v}^{[t]}(x, y)\right)}{\partial x} \\
& \times\left\{f\left(x+\bar{u}^{[t]}(x, y), y+\bar{v}^{[t]}(x, y)\right)-g(x, y)\right\}
\end{aligned}
$$




$$
\begin{array}{r}
v^{[t+1]}(x, y)=\bar{v}^{[t]}(x, y)-\frac{1}{4 \lambda} \frac{\partial f\left(x+\bar{u}^{[t]}(x, y), y+\bar{v}^{[t]}(x, y)\right)}{\partial y} \\
\times\left\{f\left(x+\bar{u}^{[t]}(x, y), y+\bar{v}^{[t]}(x, y)\right)-g(x, y)\right\},
\end{array}
$$

where the subscript $t$ stands for the number of iteration and $(\bar{u}, \bar{v})$ is an average of four neighboring $(u, v)$ 's.

We describe the coarse-to-fine strategy, which is adopted to keep its solution from being trapped by local minima and compute the displacement function $(u, v)$ efficiently. The number of layers for the coarse-to-fine strategy is assumed to be $K$ and two images in the $k$-th layer is referred as $f_{k}$ and $g_{k}$, where $1 \leq$ $k \leq K$. Note that $f_{K}$ and $g_{K}$ represent the original images of two handwritten letters. By applying the smoothing filter with size of $3 \times 3$ pixels to both of $f_{k}$ and $g_{k}$ and sampling at regular intervals, the coarser images, $f_{k-1}$ and $g_{k-1}$, are generated. The same procedure is repeated until $f_{1}$ and $g_{1}$ in the first layer are obtained. The computation of the displacement function begins at the first layer. The obtained results are used as the initial values in the next finer layer. Consequently, the displacement function $(u, v)$ with the same size of the original images is acquired.

\subsection{Normalization Procedure for Computed Displacement}

As described in Section 1 the uniqueness of the proposed method is to compute the displacement function and analyze it for handwriting verification. Since the computed displacement does not only contain the individual tendency in handwriting but also the effects of the position drift and scaling variation, we should remove such kinds of noisy effects from the computed displacement. Most of previous studies normalized the pattern on the image so as to fit the specified outer frame, while this study proposed a displacement normalization procedure that estimates the position drift and scaling variation according to the computed displacement. The details of the proposed normalization are described below.

It is assumed that $\left(u^{\prime}, v^{\prime}\right)$ is the displacement caused by handwriting $f$ 's shift drift for $g$ and that $\left(z_{x}, z_{y}\right)$ is the horizontal and vertical scaling ratio of $f$ for $g$, therefore the following equations are given,

$$
\begin{aligned}
u^{\prime} & =\frac{\sum g(x, y) u(x, y)}{n_{p}}, \\
v^{\prime} & =\frac{\sum g(x, y) v(x, y)}{n_{p}}, \\
z_{x} & =\frac{n_{p} \sum g(x, y) u(x, y) x-\sum g(x, y) x \sum g(x, y) u(x, y)}{n_{p} \sum(g(x, y) x)^{2}-\left(\sum g(x, y) x\right)^{2}} \\
z_{y} & =\frac{n_{p} \sum g(x, y) v(x, y) y-\sum g(x, y) y \sum g(x, y) v(x, y)}{n_{p} \sum(g(x, y) y)^{2}-\left(\sum g(x, y) y\right)^{2}}
\end{aligned}
$$

where $n_{p}$ is the number of pixels composing the handwriting $g$ and given as $\sum g(x, y)$. We adopted a very simple idea, that is, if $f$ has the horizontally 
$z_{x}$-times scaled shape compared with $g$, the computed horizontal displacement $u(x, y)$ should increase with the gradient of $z_{x}$ to $x$. The relationship of $z_{y}$ and $v(x, y)$ is also the same as described here.

According to the obtained $\left(u^{\prime}, v^{\prime}\right)$ and $\left(z_{x}, z_{y}\right)$, the normalized displacement function $(U, V)$ is given as

$$
\begin{aligned}
& U(x, y)=u(x, y)-u^{\prime}-z\left(x-\frac{n_{x}+1}{2}\right), \\
& V(x, y)=v(x, y)-v^{\prime}-z\left(y-\frac{n_{y}+1}{2}\right),
\end{aligned}
$$

where $z$ is the average of $z_{x}$ and $z_{y}$. It means that the 2-dimensional scaling ratio of $f$ for $g$ is determined by balancing the horizontal and vertical scaling ratio.

\subsection{Statistical Displacement Analysis for Handwriting Verification}

The method of obtaining the statistics of displacement and applying them for calculating the distance of the questionable handwriting (See Fig 1 again) are described. It is assumed that $N$ registered authentic handwritings are available in advance and that the $n^{\prime}$-th handwriting, $g_{n^{\prime}}$, is used as a standard one, where $1 \leq n^{\prime} \leq N$. The average displacement function $\left(\mu_{U}, \mu_{V}\right)$ and the variance $\left(\sigma_{U}^{2}, \sigma_{V}^{2}\right)$ are given with the following equations,

$$
\begin{aligned}
& \mu_{U}(x, y)=\frac{1}{N} \sum_{n=1}^{N} U_{n, n^{\prime}}(x, y), \\
& \mu_{V}(x, y)=\frac{1}{N} \sum_{n=1}^{N} V_{n, n^{\prime}}(x, y), \\
& \sigma_{U}^{2}(x, y)=\frac{1}{N-1} \sum_{n=1}^{N}\left(U_{n, n^{\prime}}(x, y)-\mu_{U}(x, y)\right)^{2}, \\
& \sigma_{V}^{2}(x, y)=\frac{1}{N-1} \sum_{n=1}^{N}\left(V_{n, n^{\prime}}(x, y)-\mu_{V}(x, y)\right)^{2},
\end{aligned}
$$

where $\left(U_{n, n^{\prime}}, V_{n, n^{\prime}}\right)$ is the normalized displacement function obtained in the case that the $n$-th and $n^{\prime}$-th registered handwritings are used as $f$ and $g$, respectively.

In order to verify a questionable handwriting $f$, after obtaining the normalized displacement function between $f$ and $g$, the statistical distance $d_{D A}$ is calculated with the following equation,

$$
\begin{aligned}
d_{D A} & =\sum_{x, y} \frac{g(x, y)}{\left(1-\alpha_{D A}\right)+\alpha_{D A} \sigma_{U}^{2}(x, y)}\left(U(x, y)-\mu_{U}(x, y)\right)^{2} \\
& +\sum_{x, y} \frac{g(x, y)}{\left(1-\alpha_{D A}\right)+\alpha_{D A} \sigma_{V}^{2}(x, y)}\left(V(x, y)-\mu_{V}(x, y)\right)^{2}
\end{aligned}
$$


where $\alpha_{D A}$ is a parameter controlling the effect of $\left(\sigma_{U}^{2}, \sigma_{V}^{2}\right)$. By comparing the obtained distance $d_{D A}$ with the advance-specified threshold $d_{t h}$, the questionable handwriting is judged if it is genuine or not.

\section{Simulation Results}

In order to evaluate the performance of the proposed method, a fundamental simulation was conducted. One volunteer offered twenty authentic handwritten letters for registration, $G_{r e g}=\left\{g_{r e g, 1}, \cdots, g_{r e g, 20}\right\}$, and ten genuine handwritten letters for the evaluation, $F_{\text {gen }}=\left\{f_{\text {gen }, 1}, \cdots, f_{\text {gen }, 10}\right\}$, while ten other volunteers also offered ten skillfully forged handwritten letters for the evaluation, $F_{f o g}=\left\{f_{f o g, 1}, \cdots, f_{f o g, 10}\right\}$. They were allowed to practice imitating one of the genuine handwritings and write a forged handwriting while looking at the genuine letter. Handwritings used in our experiment are a subset of the handwritten signature database produced in M. Yoshimura and I. Yoshimura's research $[9$. The size of the image was $128 \times 128$ pixels. The horizontal and vertical widths of all the handwritten letters on the image were shorter than 100 pixels. Each letter was shifted on the image so that the center of gravity could be located at the center of the image. Figure 2 illustrates the examples of a handwritten letter. It is a typical Chinese character that means 'big' or 'large' in English.

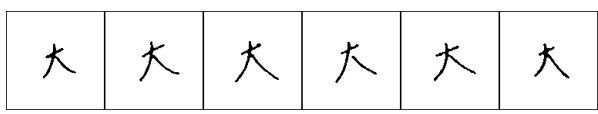

(a) registered authentic handwriting.
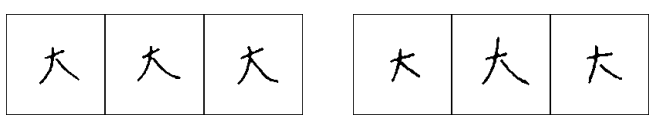

(b) genuine handwriting. (c) forged handwriting.

Fig. 2. examples of Chinese handwriting sets

First we acquired the statistics described in 2.3, that is, $\left(\mu_{U}, \mu_{V}\right)$ and $\left(\sigma_{U}^{2}\right.$, $\left.\sigma_{V}^{2}\right)$. The first registered authentic handwriting, $g_{r e g, 1}$, was used as a standard one. Figure 3(a) and (b) illustrate the standard one and the handwriting deformed with the average normalized displacement function $\left(\mu_{U}, \mu_{V}\right)$. On the other hand, Fig. 3(c) and (d) illustrate the variance $\left(\sigma_{U}^{2}, \sigma_{V}^{2}\right)$ of the normalized horizontal and vertical displacement functions, respectively, where more dark area means that its variance is larger. Note that, as shown in Eq. 16, only the statistics whose pixel is on the handwritten letter is utilized for calculating the distance $d_{D A}$.

Next the procedure for calculating the statistical distance $d_{D A}$ of a questionable handwriting is described. The regularization method with the coarse-to-fine 


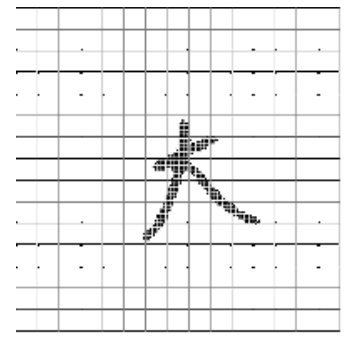

(a) standard handwriting, $g_{r e g, 1}$.

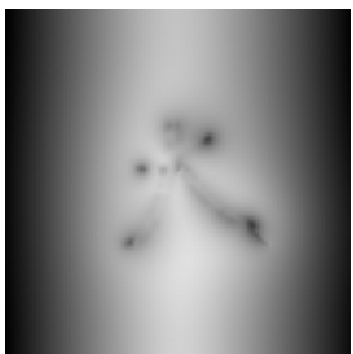

(c) variance of horizontal displacement, $\sigma_{U}^{2}$.

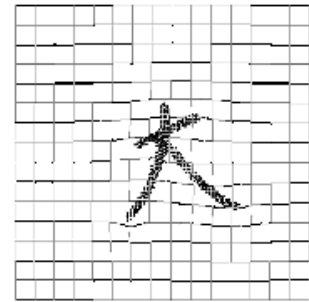

(b) average displacement, $\left(\mu_{U}, \mu_{V}\right)$.

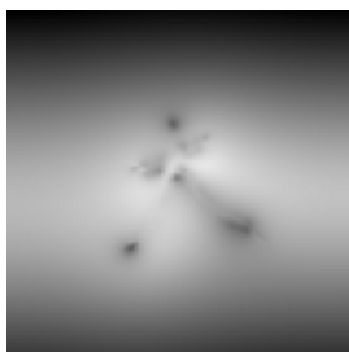

(d) variance of vertical displacement, $\sigma_{V}^{2}$.

Fig. 3. standard handwriting and displacement statistics

strategy computed the displacement function in the questionable handwriting, and then it was normalized so as to remove the noisy displacement that arose from the position drift and scaling variation. Finally, according to the displacement statistic obtained in advance from registered authentic signatures, the statistical distance $d_{D A}$ between the questionable and standard handwritings is calculated. In this simulation, $K$ was set to 5 and $\lambda$ 's were set to $5.0 \times 10^{-3}$.

Most of the previous studies evaluated the performance of the signature verification methods from the viewpoint of two types I and II error ratio, i.e., error ratio for false judgment on genuine signatures and that for false judgment on forged signatures. In this study, however, we employed the degree of separation to keep the performance evaluation from being complicated due to the configuration problem of the threshold $d_{t h}$. The degree of separation (DoS) is defined by the following equation,

$$
\eta=\frac{\left(\mu_{\text {gen }}-\mu_{\text {for }}\right)^{2}}{\sigma_{\text {gen }}^{2}+\sigma_{\text {for }}^{2}},
$$

where $\mu_{\text {gen }}$ and $\mu_{\text {for }}$ are the average of the measured distances from the standard handwriting to the genuine and forged ones, respectively. In addition, $\sigma_{g e n}^{2}$ and $\sigma_{f o r}^{2}$ are the variance to each of them, respectively.

For the comparison, the result of a conventional template matching is also shown, where the average image $\mu_{g}(x, y)$ and the variance $\sigma_{g}^{2}(x, y)$ was computed based on twenty registered authentic handwriting $g_{r e g}$ 's. This conventional method defines the distance $d_{T M}$ by the following equation, 


$$
d_{T M}=\sum_{x, y} \frac{1}{\left(1-\alpha_{T M}\right)+\alpha_{T M} \sigma_{g}^{2}(x, y)}\left(f(x, y)-\mu_{g}(x, y)\right)^{2},
$$

where $\alpha_{T M}$ is a parameter controlling the effect of $\sigma_{g}^{2}(x, y)$.

Figure 4 shows the relationships of $\alpha$ and DoS of the proposed and conventional method. In the case of $\alpha=0$, the DoSs were 2.27 and 1.61 , respectively. The proposed method gave the best DoS of 2.44 at $\alpha_{D A}=0.75$, while the conventional method gave that of 1.72 at $\alpha_{T M}=0.97$. These results implied the superiority of the proposed method.

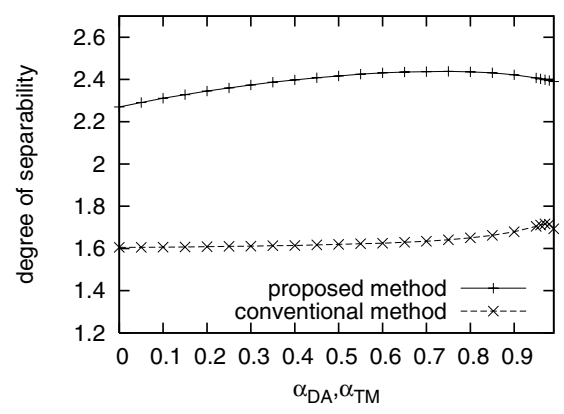

Fig. 4. degree of separation with proposed and conventional method

In order to investigate the improvement of DoS by combining the proposed method with the conventional one, the combined distance described below was introduced.

$$
d_{m i x}=(1-\beta) d_{T M}+\beta d_{D A},
$$

where $\beta$ is a parameter balancing the proposed and conventional distance.

Figure 5 illustrates the relationship between $\beta$ and DoS. The best DoS was 2.56 at $\beta=0.9$, which means that this combination can give further performance.

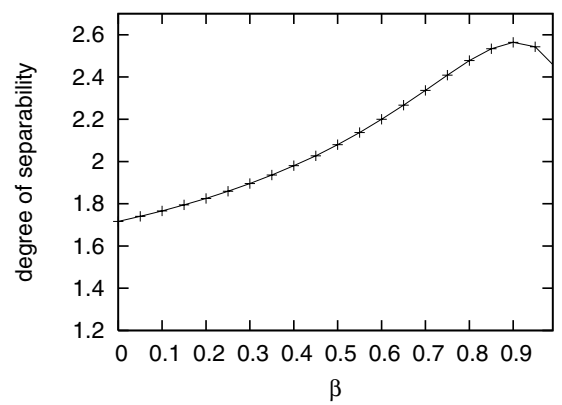

Fig. 5. degree of separation with using proposed and conventional method together 


\section{Conclusion}

In this paper, it was assumed that each writer has his or her own statistics of handwriting displacement, therefore a statistical displacement analysis for handwriting verification was proposed. Here, a regularization method with the coarse-to-fine strategy computes the displacement function in questionable handwritten letters, and then it is normalized to remove the noisy displacement that arises from the position drift and scaling variation. Finally, the normalized displacement function and the statistics of displacement obtained in advance from registered authentic letters are used to calculate the distance from a standard handwritten letter to a questionable one.

In order to evaluate the performance of the proposed method, a fundamental simulation was conducted. One volunteer offered twenty authentic handwritten letters for registration and ten genuine handwritten letters for the evaluation, while ten volunteers also offered ten skillfully forged handwritten letters for the evaluation. An indicator, degree of separation, was employed for studying how far the method separates the genuine and forged handwritings. The simulation results show that considering the statistics in handwriting displacement is effective for handwriting verification, and that the use of the proposed method with the conventional template matching method together gives further performance.

\section{References}

[1] Plamondon, R. and Lorette, G. 1989. Automatic signature verification and writer identification - the state of the art. Pattern Recognition, 22(2):107-131.

[2] de Bruyne, P. and Forré, R. 1986. Signature verification with elastic image matching. Proc. International Carnahan Conference on Security and Technology, pages $113-118$.

[3] Naske, R.-D. 1982. Writer recognition by prototype related deformation of handprinted characters. Proc. 6-th International Conference on Pattern Recognition, $2: 819-822$.

[4] Mizukami, Y., Yoshimura, M., Miike, H., and Yoshimura, I. 1999. An off-line signature verification system using an extracted displacement function. Proc. 5th ICDAR, 1:757-760.

[5] Poggio, T., Torre, V., and Koch, C. 1985. Computational vision and regularization theory. NATURE, 317(6035):314-319.

[6] March, R. 1988. Computation of stereo disparity using regularization. Pat. Recog. Let., 8(3):181-188.

[7] Mizukami, Y. 1998. A handwritten Chinese character recognition system using hierarchical displacement extraction based on directional features. Pattern Recognition Letters, 19(7):595-604.

[8] Horn, B. and Schunck, B. 1981. Determining optical flow. Artificial Intelligence, 17:185-203.

[9] Yoshimura, I. and Yoshimura, M. 1994. Off-line verification of Japanese signature after elimination of background patterns. International Journal of Pattern Recognition and Artificial Intelligence, 8(3):693-708. 